\title{
Acute headache in general neurology of China: Cause changes and predictors of short-term outcome*
}

\author{
Guangsheng Wang ${ }^{1}$, Yeting Zhou ${ }^{2}$, Xiaodong Chen ${ }^{1}$, Hongjian Wang ${ }^{3}$, Tonghui Yang ${ }^{1}$, \\ Chunhong Chang ${ }^{1}$, Yuanwei Wang ${ }^{1}$, Hanpei Gu ${ }^{1}$, Daoming Tong ${ }^{1 \#}$ \\ ${ }^{1}$ Departments of Neurology, Shuyang People's Hospital, Shuyang, China \\ ${ }^{2}$ Medical Evaluation Unit, Shuyang People's Hospital, Shuyang, China \\ ${ }^{3}$ Department of Medical Affairs, Shuyang People's Hospital, Shuyang, China \\ Email: "tongdaoming@163.com
}

Received 16 January 2012; revised 17 February 2012; accepted 21 February 2012

\begin{abstract}
Background: Although patients with acute headache frequently present in emergency department, the causes and predictors of short-term outcome in patients with acute headache in general neurology have not been adequately investigated. Methods: We prospectively reviewed the medical records of 130 hospitalized acute headache patients in general neurology of China. Their initial CT scan was assessed, as was their lumbar puncture (LP) examination if performed. Results: The main cause of acute headache was acute secondary headache $(80.8 \%)$, which was mainly attributable to acute cerebrovascular events (72.4\%) followed by intracranial infection (19.0\%). Among the $10.8 \%$ of patients who died during hospitalization most $(85.7 \%)$ had subarachnoid hemorrhage (SAH). Significant predictors of survival were severe headache versus thunderclap headache, meningismus, delayed loss of consciousness, and hypertension (all $p<0.05$ ). Multiple logistic regression analyses showed significant differences in severe headache or thunder-clap headache (OR, $0.255 ; 95 \% \mathrm{CI}, 0.066-0.990 ; p=0.048$ ) and delayed loss of consciousness (OR, 0.060; $95 \% \mathrm{CI}$, $0.016-0.224, p=0.000$ ) between patients who died and those who survived. Conclusions: The main underlying cause of hospitalized acute headache was acute cerebrovascular events. Severe headache and delayed loss of consciousness are predictors for poor outcome of acute headache.
\end{abstract}

Keywords: Acute Headache; Causes; Cerebrovescular Events; Subarachnoid Hemorrhage; Outcome

\section{INTRODUCTION}

Acute headache (primary and secondary) is a common

\footnotetext{
${ }^{*}$ The authors declare that they have no competing interests.

\#Corresponding author.
}

neurological emergency that may be caused by a great variety of clinical conditions. However, the underlying causes may change pattern over time particularly where there is rapid social development. Clinical study of acute headache presenting in emergency departments has been previously reported [1-5]. Many acute secondary headaches can be misdiagnosed if appropriate investigations are not made, even life-threatening subarachnoid hemorrhage (SAH) [6-8]. However, there are few reports on patients with acute headache who are hospitalized at general department of neurology [9]. The aims of the study were to investigate underlying causes of acute headache and to determine predictors of outcome in patients with acute headache.

\section{METHODS}

Consecutive patients with acute headache admitted either directly or through the emergency department into a general department of neurology at a public hospital in China from February 2003 to January 2006 were prospectively evaluated. To be eligible, patients had to have what they described as an acute onset of sudden or severe headache. Acute headache was defined as a headache of acute or sudden onset and lasting several hours, which without the history of headache, or with the history of headache but no onset of headache at least over a > 2-month period. Severe headache was defined as worst of their lives or a significant lifestyle restriction. Thunderclap headache was defined as a sudden and severe headache with maximum intensity at onset. Patients with delayed loss of consciousness were accepted if they were initially alert at the onset of headache. Exclusion criteria included age $<15$ years, recent head trauma or lumbar puncture (LP), initial loss of consciousness, signs of hemiplegia, and history of $\geq 3$ recurrent headaches of the same character and intensity as the presenting headache over a $>6$-month period. The study was approved by the Ethical Committee on Clinical Research of the hospital; 
informed consent was obtained from all patients or their families.

On admission, the patients responded to an approved questionnaire providing data on sex and age, prior headache ictus, and family history. All patients were examined by neurologist for clinical and full neurological states, especially meningismus, within $24 \mathrm{~h}$ from admission.

Computed tomography (CT) scan was performed by all patients within $24 \mathrm{~h}$ of admission to hospital. Following this procedure, patients with negative CT and suspected SAH or intracranial infection underwent LP examination. CSF was analyzed for white blood cell count and protein and glucose levels. Spectrophotometry for detection of oxyhemoglobin was also performed for these patients. Staining for acid-fast bacilli and a test for cryptococcal antigen were investigated.

In evaluation, case histories and test data were assessed by two experienced neurologists; separate diagnoses were made following guidelines of the International Classification of Headache Disorders (ICHD) [10].

\section{Statistical Methods}

Data are presented as mean $\pm \mathrm{SD}$ or $\mathrm{n}(\%)$. Frequencies were compared by Chi-squared statistics or Fisher's exact test as appropriate. Continuous variables were compared by $t$ test. Patient survival was assessed by univariate analysis. Predictors of outcome were identified with forward stepwise logistic regression. Differences with $p$-value $<0.05$ were considered significant. All statistical analyses were performed using SPSS version 10.0 software (SPSS Inc., Chicago, IL, USA).

\section{RESULTS}

\subsection{Diagnosis of Acute Headache}

A total of 130 consecutive patients (M/F, 54/64; age range, 15 - 72 years) with acute headache were enrolled, which were admitted either directly to the hospital or through the emergency department. The interval from headache onset to admission in the majority of the patients was $<24 \mathrm{~h}$; in a few patients this was $>1$ week due to delayed referral from general practitioner. Shortest and longest length of hospitalization was 6 and 62 days, respectively; average stay was $18 \pm 11$ days.

Although many patients $(n=25 ; 19.2 \%)$ had a primary headache (all acute migraine) most cases were acute secondary headache $(\mathrm{n}=105 ; 80.8 \%)$, among whom acute cerebrovascular events were noted in the majority (72.4\%). Characteristics of 130 patients with acute headacheare shown in Table 1.

Results of CT and LP evaluations are displayed in Table 2 . In the positive CT group 73 patients $(69.5 \%)$ were diagnosed, of whom 47 patients had SAH (64.4\%), 15
Table 1. Characteristics of 130 patients with acute headache.

\begin{tabular}{lr}
\hline Characteristics & No of patients \\
\hline Sex(male/f, \%) & $60(46.2 \%)$ \\
Age range (years) & $15-72$ \\
The times from headache onset to admission $<24$ hours & $107(82.3 \%)$ \\
The times from headache onset to admission $<2$ weeks & $23(17.7 \%)$ \\
Sudden headache & $78(60.0 \%)$ \\
Severe headache or thunderclap headache & $55(42.3 \%)$ \\
Meningismus & $68(52.3 \%)$ \\
Vomiting & $48(36.9 \%)$ \\
Fiver & $16(12.3 \%)$ \\
Delayed loss of consciousness & $25(19.2 \%)$ \\
Hypertension & $27(20.8 \%)$ \\
length of hospitalization (days, mean \pm SD) & $18 \pm 11$ \\
Diagnostic procedures & \\
Computed tomography & $14(10.8 \%)$ \\
Both computed tomography and lumbar puncture & $130(100 \%)$ \\
Final diagnosis and 30 days outcome & $39(30.0 \%)$ \\
Acute migraine & \\
Subarachnoid haemorrhage & $3(2.3 \%)$ \\
Intracerebral hemorrhage & $25(19.2 \%)$ \\
Lacuna infacts & $54(41.5 \%)$ \\
Hypertension encephalopathy & $15(11.5 \%)$ \\
Brain tumour & $3(2.3 \%)$ \\
\hline
\end{tabular}

Table 2. Diagnoses of CT and LP in patients with acute headache.

\begin{tabular}{cccc}
\hline Diagnoses & $\begin{array}{c}\text { Positive CT group } \\
(\mathrm{n}=73)\end{array}$ & $\begin{array}{c}\text { Negative CT group } \\
(\mathrm{n}=57)\end{array}$ & $P$ Value \\
\hline SAH & $47(64.4 \%)$ & $7(12.3 \%)$ & 0.000 \\
$\begin{array}{c}\text { Intracerebral } \\
\text { hemorrhage }\end{array}$ & $15(20.5 \%)$ & $0(0)$ & 0.000 \\
Lacuna infacts & $3(4.1 \%)$ & $0(0)$ & 0.122 \\
Brain tumour & $4(5.5 \%)$ & $0(0)$ & 0.073 \\
$\begin{array}{c}\text { Intracranial } \\
\text { infections }\end{array}$ & $3(4.1 \%)$ & $17(29.8 \%)$ & 0.000 \\
Hydrocephalus & $1(1.4 \%)$ & $0(0)$ & 0.375 \\
Others & $0(0)$ & $33(57.9 \%)$ & 0.000 \\
\hline
\end{tabular}


intracerebral hemorrhage $(20.5 \%$; 7 cases lobar, 4 cerebellar, 1 thalamic, 3 primary intraventricular), 3 lacunar infarction $(4.1 \%$; 2 cases internal capsule, 1 multiple lacunar in white matter; age range, 49 - 61 years), and 3 intracranial infection $(4.1 \%$; 1 case viral encephalitis, 1 tuberculosis encephalitis, 1 unknown encephalitis; all diagnosed by LP).

The negative CT group included 57 patients in whom LP was performed in 31 cases (54.4\%); in these individuals 7 patients with $\mathrm{SAH}$ were diagnosed by oxyhemoglobin CSF $(12.3 \%$; 6 cases by LP within 1 week of onset and 1 within 13 days, 2 of whom died). We diagnosed 17 intracranial infections $(29.8 \%$; 15 patients CSF positive and 2 CSF negative) including 5 cases of tuberculous meningitis, 6 viral meningitis, and 6 viral encephalitis.

\subsection{Univariate and Multivariate Analysis of Outcome}

Although 116 of 130 patients (89.2\%) had a good outcome, 14 of 130 patients $(10.8 \%)$ died during hospitalization, among whom the majority (12 cases; $85.7 \%$ ) had SAH (of those, $75 \%$ died of rebleed); 1 patient (7\%) died of fourth ventricle hemorrhage and $1(7 \%)$ brain tumor.

Univariate analysis of death in our patients is summarized in Table 3. There was no difference in sex, age, sudden headache, vomiting, and fever in survival patients and those who died $(p>0.05)$. However, there were significant differences in headache versus thunderclap headache, meningismus, delayed loss of consciousness, and hypertension in the survival and non-survival group $(p<0.05)$.

Table 4 summarizes the results of multiple logistic regressions based on four data of the original six variables. Significant predictors of death were severe versus thunderclap headache (OR, 0.255; 95\%CI, 0.066 - 0.990;

Table 3. The results of univariate analysis in survival and non-survival patients.

\begin{tabular}{cccc}
\hline Variables & $\begin{array}{c}\text { Survival } \\
(\mathrm{n}=116)\end{array}$ & $\begin{array}{c}\text { Non-survival } \\
(\mathrm{n}=14)\end{array}$ & $P$ Value \\
\hline Sex(male/f, \%) & $54 / 62$ & $6 / 8$ & 0.793 \\
Age(y, mean $\pm \mathrm{SD})$ & $44.7 \pm 15.3$ & $51.9 \pm 10.5$ & 0.090 \\
$\begin{array}{c}\text { Sudden headache } \\
\text { Severe or thunderclap } \\
\text { headache }\end{array}$ & $69(59.5 \%)$ & $9(64.3 \%)$ & 0.234 \\
$\begin{array}{c}\text { Meningismus } \\
\text { Vomiting }\end{array}$ & $57(38.8 \%)$ & $10(71.4 \%)$ & 0.020 \\
Fiver & $40(34.5 \%)$ & $8(57.1 \%)$ & 0.097 \\
$\begin{array}{c}\text { Delayed loss of } \\
\text { consciousness }\end{array}$ & $14(13.4 \%)$ & $2(14.3 \%)$ & 0.812 \\
Hypertension & $21(18.1 \%)$ & $6(42.9 \%)$ & 0.031 \\
\hline
\end{tabular}

Table 4. Results analyzed by the multiple Logistic regression for predictors of outcome in patients with acute headache.

\begin{tabular}{|c|c|c|c|c|c|c|}
\hline Variables & B & SE & Wald & $P$ Value & OR & $95 \% \mathrm{CI}$ \\
\hline $\begin{array}{l}\text { Severe or } \\
\text { thunderclap } \\
\text { headache }\end{array}$ & -1.366 & 0.692 & 17.480 & 0.048 & 0.255 & $\begin{array}{c}0.066 \sim \\
0.990\end{array}$ \\
\hline $\begin{array}{l}\text { Delayed loss } \\
\text { of } \\
\text { consciousness }\end{array}$ & -2.820 & 0.674 & 3.900 & 0.000 & 0.060 & $\begin{array}{c}0.016 \sim \\
0.224\end{array}$ \\
\hline Constant & 3.986 & 0.707 & 31.770 & 0.000 & 53.835 & \\
\hline
\end{tabular}

$p=0.048$ ), and delayed loss of consciousness (OR, $0.060 ; 95 \% \mathrm{CI}, 0.016-0.224, p=0.000$ ) between the two groups.

\section{DISCUSSION}

In our 130 patients diagnosed according to ICHD standards secondary headache was the major cause of acute headache for hospitalization, in $80.8 \%$, including acute cerebrovascular events in $72.4 \%$, intracranial infection in $19.0 \%$, and a few other causes. Acute primary headache only accounted for $19.2 \%$ of those hospitalized; all were acute migraine. This clinical study clearly indicates that in patients with acute headache referred for hospital treatment acute cerebrovascular events should be foremost borne in mind.

On the other hand, however, in our cohort the proportion with secondary headache and acute cerebrovascular events was significantly higher than previously observed in emergent patients in neurology departments reported in Canada and Norway [2,3], and cerebrovascular events were significantly higher than in patients hospitalized in Minnesota [9]. The present study shows that the pattern of causes for acute headache is not the same in emergency room and hospitalized patients, and may differ according to region, particularly the incidence of acute cerebrovascular events were higher in China than in western countries [11-13].

SAH is a common cause of acute secondary headache in emergency settings [1-6]. The present data demonstrated that SAH is also common cause of acute seconddary headache in hospitalized patients. The probability of detecting SAH is proportional to the clinical grade and time from the event [14]. In the first $12 \mathrm{~h}$ after SAH, the sensitivity of CT for SAH is $98 \%$ [15], declining to $93 \%$ at $24 \mathrm{~h} \mathrm{[4]}$ and $85 \%$ at 6 days after SAH [16]. In our 54 patients with acute headache from SAH most $(87.0 \%)$ were diagnosed by CT scan, similar to the rate in the literature. We also showed that the probability of patients with acute headache attributed to $\mathrm{SAH}$ with a negative CT scan within 2 weeks of onset was $12.3 \%$. This is similar to the report of O'Weill et al. [17], in which patients with acute headache with SAH in the negative CT 
group accounted for $15 \%-24 \%$. However, we have to point out that current proportion may be with potential limitations, as those SAH with initial loss of consciousness were not included.

Our study suggests that LP should be carried out in patients with suspected SAH and negative results on head CT [7] especially those with low Hunt and Hess grade on admission and normal diastolic blood pressure; those with suspected SAH but delayed CT scan should undergo LP [18] so as not to miss the most life-threatening diagnosis of SAH.

Our data show that acute lacunar infarction was also causative of acute secondary headache. Arboix et al. [19] noted that in patients with lacunar infarction, headache at stroke onset was more common in deep brain gray matter or brainstem topographies than in supratentorial white matter lesions. However, in our 3 patients with lacunar infarcts the lesions were located in the supratentorial white matter, suggesting that lacunar infarct in supratentorial white matter may also cause acute headache.

Acute primary headache in hospitalized patients was mainly migraine headache. Because most of our patients experienced first severe sudden attack of migraine, they were diagnosed as acute migraine with or without aura. This was similar to the pattern noted of Bø, S.H. et al. [3].

In this study mortality of patients with acute headache during hospitalization was $10.8 \%$. In these individuals SAH accounted for $85.7 \%$ of total deaths, including 2 patients who died with a negative CT scan and 1 oxyhemoglobin confirmed by LP. Our study showed that $\mathrm{SAH}$ is the most life-threatening acute secondary headache with a mortality of $75 \%$ for persons who rebleed, consistent with reported guidelines [14]. Moreover, we confirmed that sudden, severe headache rather than thunderclap headache, meningeal irritation, delayed loss of consciousness, and hypertension in the non- survival group were significantly higher than in survivors, which are so-called "red flag" symptoms as previously reported $[20,21]$. On the other hand, multiple logistic regression analysis of our data revealed that severe head- ache and delayed loss of consciousness were significantly and independently related to poor outcome in acute headache.

We observed markedly higher percent hemorrhagic stroke than that reported in Western countries [22]. The high mortality of acute headache may be not only associated with higher percent hemorrhagic stroke, but also with higher mortality of hemorrhagic strokes [23], particularly SAH [14].

In conclusion, the main underlying cause of hospitalized acute headache was acute cerebrovascular events. Severe headache and delayed loss of consciousness are predictors for poor outcome of acute headache. Therefore, we believe that a better understanding of the causes changes of acute headache and predictors of outcome for acute headache patients would facilitate design of protocols describing its differential diagnosis and early intervention.

\section{REFERENCES}

[1] Landtblom, A.M., Fridriksson, S., Boivie, J., Hillman, J., Johansson, G. and Johansson, I. (2002) Sudden onset headache: A prospective study of features, incidence and causes. Cephalalgia, 22, 354-360. doi:10.1046/j.1468-2982.2002.00368.x

[2] Perry, J.J., Stiell, I.G., Wells, G.A., et al. (2005) Attitudes and judgment of emergency physicians in the management of patients with acute headache. Academic Emergency Medicine, 12, 33-37. doi:10.1111/j.1553-2712.2005.tb01475.x

[3] Bø, S.H., Davidsen, E.M., Gulbrandsen, P. and Dietrichs, E. (2008) Acute headache: A prospective diagnostic work-up of patients admitted to a general hospital. European Journal of Neurology, 15, 1293-1299. doi:10.1111/j.1468-1331.2008.02279.x

[4] Morgenstern, L.B., Luna-Gonzales, H., Huber, J.C. Jr., et al. (1998) Worst headache and subarachnoid hemorrhage: Prospective, modern computed tomography and spinal fluid analysis. Annals of Emergency Medicine, 32, $297-$ 304.

[5] Grimaldi, D., Nonino, F., Cevoli, S., Vandelli, A., D'Amico, R. and Cortelli, P. (2009) Risk stratification of non-traumatic headache in the emergency department. Journal of Neurology, 256, 51-57. doi:10.1007/s00415-009-0033-0

[6] Vermeulen, M.J. and Schull, M.J. (2007) Missed diagnosis of subarachnoid hemorrhage in the emergency department. Stroke, 38, 1216-1221. doi:10.1161/01.STR.0000259661.05525.9a

[7] Suarez, J.I., Tarr, R.W. and Selman, W.R. (2006) Aneurysmal subarachnoid hemorrhage. New England Journal of Medicine, 354, 387-396. doi:10.1056/NEJMra052732

[8] Kowalski, R.G., Claassen, J., Kreiter, K.T., Bates, J.E., Ostapkovich, N.D., Connolly, E.S. and Mayer, S.A. (2004) Initial misdiagnosis and outcome after subarachnoid hemorrhage. The Journal of the American Medical Association (JAMA), 291, 866-869.

doi:10.1001/jama.291.7.866

[9] Ramirez-Lassepas, M., Espinosa, C.E., Cicero, J.J., Johnston, K.L., Cipolle, R.J. and Barber, D.L. (1997) Predictors of intracranial pathologic findings in patients who seek emergency care because of headache. Archives of Neurology, 54, 1506-1509. doi:10.1001/archneur.1997.00550240058013

[10] Headache Classification Subcommittee of the International Headache Society (2004) The international classification of headache disorders, 2nd edition. Cephalalgia, 24, 1-160.

[11] Thorvaldsen, P., Asplund, K., Kuulasmaa, K., Rajakangas, A.M. and Schroll, M. (1995) Stroke incidence, case fatality, and mortality in the WHO MONICA project. World 
health organization monitoring trends and determinants in cardiovascular disease. Stroke, 26, 361-367.

[12] Xu, G., Yin, Q. and Liu, X. (2009) Narrowing the gap of stroke management between developed and developing countries: Report of the fifth international stroke summit, Nanjing, China, July 17-19, 2009. Cerebrovascular Diseases, 29, 101-102. doi:10.1159/000259620

[13] Ferri, C.P., Schoenborn, C., Kalra, L., Acosta, D., Guerra, M., Huang, Y., et al. (2011) Prevalence of stroke and related burden among older people living in Latin America, India and China. Journal of Neurology, Neurosurgery, and Psychiatry, 82, 1074-1082. doi:10.1136/jnnp.2010.234153

[14] Bederson, J.B., Connolly, E.S. Jr., Batjer, H.H., Dacey, R.G., Dion, J.E., Diringer, M.N., et al. (2009) Guidelines for the management of aneurysmal subarachnoid hemorrhage: A statement for healthcare professionals from a special writing group of the stroke council, American heart association. Stroke, 40, 994-1025. doi:10.1161/STROKEAHA.108.191395

[15] Van der Wee, N., Rinkel, G.J.E., Hasan, D. and van Gijn, J. (1995) Detection of subarachnoid haemorrhage on early CT: Is lumbar puncture still needed after a negative scan? Journal of Neurology, Neurosurgery, and Psychiatry, 58, 357-359. doi:10.1136/jnnp.58.3.357

[16] Van Gijn, J. and van Dongen, K.J. (1982) The time course of aneurysmal haemorrhage on computed tomo- grams. Neuroradiology, 23, 153-156.

[17] O'Weill, J., Mclaggan, S. and Gibson, R. (2005) Acute headache and subarachnoid heamorrhage: A retrospective review of CT and lumbar puncture finding. Scottish Medical Journal, 50, 151-153.

[18] Tong, D.M. and Chou, Y.T. (2010) Predictors of the subarachnoid hemorrhage of a negative CT scan. Stroke, 41, e566-e567. doi:10.1161/STROKEAHA.109.574038

[19] Arboix, A., Grau-Olivares, M., Garcia-Eroles, L., et al. (2006) Clinical implications of headache in lacunar stroke: Relevance of site of infarct. Headache, 46, 1172-1180. doi:10.1111/j.1526-4610.2006.00507.x

[20] Breen, D.P., Duncan, G.W., Pope, A.E., Gray, A.J. and Al-Shahi, S.R. (2008) Emergency department evaluation of sudden, severe headache. QJM: An International Journal of Medicine, 101, 435-443. doi:10.1093/qimed/hen036

[21] Clinch, C.R. (2001) Evaluation of acute headaches in adults. American Family Physician, 63, 685-692.

[22] Jia, Q., Liu, L.P. and Wang, Y.J. (2010) Stroke in China. Clinical and Experimental Pharmacology and Physiology, 37, 259-264. doi:10.1111/j.1440-1681.2009.05290.x

[23] Jiang, Y., Li, X.Y., Hu, N., Huang, Z.J. and Wu, F. (2010) Epidemiologic characteristics of cerebrovascular disease mortality in China, 2004-2005. Zhonghua Yu Fang Yi Xue Za Zhi, 44, 293-297. 